\title{
Protective effect of alamandine on doxorubicin-induced nephrotoxicity in rats
}

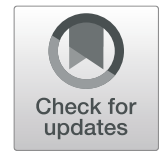

Ava Soltani Hekmat, Ameneh Chenari, Hiva Alipanah and Kazem Javanmardi ${ }^{*}$

\begin{abstract}
Background: This study aimed to evaluate the protective effects of alamandine, a new member of the angiotensin family, against doxorubicin (DOX)-induced nephrotoxicity in rats.

Methods: Rats were intraperitoneally injected with DOX $(3.750 \mathrm{mg} / \mathrm{kg} /$ week) to reach a total cumulative dose of $15 \mathrm{mg} / \mathrm{kg}$ by day 35 . Alamandine ( $50 \mu \mathrm{g} / \mathrm{kg} / \mathrm{day})$ was administered to the rats via mini-osmotic pumps for 42 days. At the end of the experiment, rats were placed in the metabolic cages for $24 \mathrm{~h}$ so that their water intake and urine output could be measured. After scarification, the rats' serum and kidney tissues were collected, and biochemical, histopathological, and immunohistochemical studies were carried out.
\end{abstract}

Results: DOX administration yielded increases in pro-inflammatory cytokines, including interleukin (IL)-1 $\beta$ and IL-6, pro-fibrotic proteins transforming growth factor- $\beta$ (TGF- $\beta$ ), pro-inflammatory transcription factor nuclear kappa $B$ (NF-KB), kidney malondialdehyde (MDA), creatinine clearance, blood urea nitrogen (BUN), and water intake. On the other hand, the DOX-treated group exhibited decreased renal superoxide dismutase (SOD), renal glutathione peroxidase (GPx) activity, and urinary output. Alamandine co-therapy decreased these effects, as confirmed by histopathology and immunohistochemical analysis.

Conclusions: The results suggest that alamandine can prevent nephrotoxicity induced by DOX in rats.

Keywords: Alamandine, Doxorubicin, Kidney, Cytokine, Oxidative Stress, Apoptosis, Immunohistochemistry

\section{Introduction}

Doxorubicin (DOX) is an antibiotic anthracycline that was first isolated in 1960 and has been used for the past 30 years to treat cancer patients [1]. DOX has several side effects, such as cardiac and renal toxicity. DOX-mediated nephropathy is caused by glomeruli destruction and tubule damage [2, 3]. DOX-induced nephrotoxicity has been studied much less than other anthracycline-related side effects. DOX induces nephropathy via a complex mechanism. Free radicals, lipid peroxidation, and decreased antioxidant enzyme activity are among the most likely primary mediators in developing nephrotic syndrome $[3,4]$. Inflammation also plays a significant role in DOX-induced

\footnotetext{
* Correspondence: kjavanmardi@fums.ac.ir

Department of Physiology, Fasa University of Medical Sciences, Ebn-E-Sina $\mathrm{SQ}$, Fasa, Iran
}

kidney damage via the effects of topical cytokines and other cytotoxic factors [2]. DOX can also cause proteins to be excreted in the urine by destroying the nephrons directly.

DOX-induced nephropathy is a classic model of renal failure in rats. Damage to the filtration barrier is the primary cause of protein excretion due to renal failure [2]. DOX is responsible for this filtration barrier damage $[5,6]$.

Thus, administering drugs with anti-inflammatory and antioxidant effects could mitigate the side effects of DOX. Alamandine is a new member of the reninangiotensin system (RAS) family that protects the cardiovascular system and kidney functions [7].

Alamandine, like angiotensin (1-7) [Ang (1-7)], is a heptapeptide. It differs from Ang (1-7) in only one amino acid in the N-terminal region. It exerts its effects by binding to the Mas-related G-protein coupled

(c) The Author(s). 2021 Open Access This article is licensed under a Creative Commons Attribution 4.0 International License, which permits use, sharing, adaptation, distribution and reproduction in any medium or format, as long as you give appropriate credit to the original author(s) and the source, provide a link to the Creative Commons licence, and indicate if changes were made. The images or other third party material in this article are included in the article's Creative Commons licence, unless indicated otherwise in a credit line to the material. If material is not included in the article's Creative Commons licence and your intended use is not permitted by statutory regulation or exceeds the permitted use, you will need to obtain permission directly from the copyright holder. To view a copy of this licence, visit http://creativecommons.org/licenses/by/4.0/. The Creative Commons Public Domain Dedication waiver (http://creativecommons.org/publicdomain/zero/1.0/) applies to the data made available in this article, unless otherwise stated in a credit line to the data. 
receptor of the type $\mathrm{D}(\mathrm{MrgD})$ receptor[8-10]. The renal actions of Ang (1-7 (might be related to reductions in oxidative stress and the activation of anti-apoptotic pathways [11].

Lu et al. showed that Ang (1-7) protects kidneys from injuries caused by hypoxia by reducing oxidative stress, fibrosis, and inflammation [11]. Also, Mori et al. used a model of diabetic nephropathy to show that Ang (1-7) can protect the kidneys by reducing inflammation, oxidative stress, and lipotoxicity [12]. In addition, it was recently reported that Ang (1-7) reduces DOX-induced cardiotoxicity in rats through antioxidant mechanisms[13].

Alamandine also has anti-fibrotic and antiinflammatory effects [7, 14]. For example, alamandine increases antioxidant expression in ventricles exposed to ischemia-reperfusion injury[15].Moreover, alamandine reduces pro-inflammatory factors induced by aortic constriction, such as TNF- $\alpha$ and IL-1 $\alpha$, in mice [16]. In a study on an animal model of sepsis induction by polysaccharides in C57BL6/J mice, plasma and tissue levels of interleukin-1B (IL-1B) and tumor necrosis factor alpha (TNFo) increased. At the same time, alamandine reduced the levels of inflammatory cytokines and apoptosis in cardiac tissues [17]. To the best of our knowledge, no prior studies have examined the effects of alamandine on renal function and DOX-induced nephrotoxicity.

Many experimental and clinical reports have highlighted the protective role of Ang (1-7) in renal hemodynamics and functions under different conditions [18]. Also, alamandine has similar effects to Ang (1-7) $[9,19]$. Therefore, we assumed that alamandine protects against DOX-induced nephrotoxicity.

In this study, several biomarkers of renal injury were measured in DOX-treated rats and alamandine/DOX coadministrated rats. These biomarkers included blood urea nitrogen (BUN); creatinine; creatinine clearance; serum urea; albumin; and pro-inflammatory cytokines, including IL-1 $\beta$ and IL-6, pro-inflammatory transcription factor nuclear kappa B (NF- $\mathrm{B} B$ ), pro-fibrotic proteins transforming growth factor- $\beta$ (TGF- $\beta$ ), and renal antioxidant enzymes [superoxide dismutase (SOD), glutathione peroxidase (GPx), and malondialdehyde (MDA)]. Histological and immunohistochemistry analyses were also performed.

\section{Materials and methods Materials}

This experiment was performed on 35 male SpragueDawley rats (180-220 g) obtained from the Experimental Animal Centre of Fasa University of Medical Sciences. All procedures followed relevant guidelines and regulations regarding the care and use of animals for the experimental procedures. The procedures were also approved by the Committee of Animal Care of the Fasa University of Medical Sciences (IR.FUMS.REC.1397.014) in compliance with the ARRIVE guidelines [20]. All animals were acclimatized under the controlled standard conditions of $12 \mathrm{~h}$ light/12 $\mathrm{h}$ dark cycles, at a temperature of $20-22{ }^{\circ} \mathrm{C}$, standard pellet diets, and water ad libitum for one week before the start of experiments. Alamandine and DOX were obtained from Phoenix Pharmaceuticals Inc., CA, USA, and Tocris Bioscience, respectively.

\section{Experimental group design}

After one week of acclimatization to cages, the rats were randomly divided into five groups:

1. The control group comprised intact animals that experienced no surgical interventions and were given no drugs.

2. The sham group received normal saline as a vehicle for 42 days via mini-osmotic pumps (model 2006;

ALZET Osmotic Pumps, CA, USA) that were surgically placed subcutaneously between the scapulae. This group also received normal saline intraperitoneal (i.p) on days 14, 21, 28, and 35.

3. The DOX group received DOX dissolved in normal saline $(3.750 \mathrm{mg} / \mathrm{kg})$ i.p on days $14,21,28$, and 35 to reach the total cumulative dose $(15 \mathrm{mg} / \mathrm{kg})$.

4. The alamandine group received alamandine dissolved in normal saline for 42 days by mini-osmotic pumps with an infusion rate of $0.15 \mu \mathrm{l} / \mathrm{h}(50 \mu \mathrm{g}$ alamandine/ $\mathrm{kg} /$ day) as described by Liu et al. [21].

5. The DOX + alamandine group received alamandine by mini-osmotic pumps for 42 days $(50 \mu \mathrm{g}$ alamandine $/ \mathrm{kg} /$ day $)$ and DOX $(3.750 \mathrm{mg} / \mathrm{kg})$ i.p on days $14,21,28$, and 35 to reach the total cumulative dose $(15 \mathrm{mg} / \mathrm{kg})$ as described by Warp et al. [22].

On day 41, rats were housed in the individual metabolic cages. A 24-h urine sample was collected from each animal so that the creatinine clearance, creatinine, albumin, and TGF-B levels could be measured. On day 42 (seven days after the final DOX injection), the animals were euthanized with pentobarbital sodium (150 mg/kg, intraperitoneal, IP). Blood samples were taken, and both kidneys were removed from all rats. The blood samples were centrifuged at $4000 \mathrm{rpm}$ for $10 \mathrm{~min}$. Serums, which were used to evaluate biochemical parameters, were taken and maintained at $-80{ }^{\circ} \mathrm{C}$. Creatinine clearance (used to estimate the glomerular filtration rate (GFR)) was determined as described by Iyalomhe et al. [23] and Ogundipe et al. [24] This was done based on the 24-h urine samples obtained under the following formula (expressed as $\mathrm{ml} / \mathrm{min}$ ): Creatinine clearance 
$(\mathrm{ml} / \mathrm{min})=\mathrm{mg}$ creatinine $/ \mathrm{dl}$ urine $\times \mathrm{ml}$ urine $24 \mathrm{~h} / \mathrm{mg}$ creatinine/dl serum $\times 1440$.

Rats' right kidneys were dissected and washed with PBS (10 mM PO43-, $137 \mathrm{mM} \mathrm{NaCl}$, and $2.7 \mathrm{mM} \mathrm{KCl}$; $\mathrm{pH}=7.4)$. They were then dried on filter paper and weighed. Afterward, they were homogenized in the PBS and centrifuged at $10,000 \times \mathrm{g}$ for $20 \mathrm{~min}$ at $4{ }^{\circ} \mathrm{C}$. The supernatants were stored at $-20{ }^{\circ} \mathrm{C}$ until the analyses of oxidative stress parameters, antioxidants, and TGF- $\beta$. These analyses were carried out using Eliza kits.

\section{Assessment of Water Intake and urine volume}

Water intake and urine volume were assessed using metabolic cages. Water intake was calculated as the difference between the final volume measured and the remaining volume over $24 \mathrm{~h}$. This measurement was considered as the daily water intake for every animal in each experimental group.

The volumes for both water intake and urine output were measured using measuring cylinders.

\section{Assessment of inflammatory cytokines in serum}

The IL-6 assay kit (cat. no. SEA079Ra), NF-kB assay kit (cat. no. SEB824Ra), and IL-1 $\beta$ assay kit (cat. no. SEA563Ra) were purchased from Cloud-Clone Corp (Cloud-Clone Corp Technology co., Ltd., Wuhan, China). Briefly, for IL-6 assay, $100 \mu \mathrm{l}$ of a standard blank or samples was added into the appropriate wells and then incubated for $1 \mathrm{~h}$ at $37{ }^{\circ} \mathrm{C}$. After aspiration, $100 \mu \mathrm{l}$ of prepared detection reagent was added to each well and incubated for $1 \mathrm{~h}$ at $37{ }^{\circ} \mathrm{C}$. After aspiration and washing, $90 \mu \mathrm{L}$ of substrate solution was added to wells and incubated for $15 \mathrm{~min}$ at $37^{\circ} \mathrm{C}$. Finally, $50 \mu \mathrm{L}$ of stop solution was added, and OD was immediately measured with a BioTek ELISA reader at $450 \mathrm{~nm}$. The IL-6 concentration was determined by comparing the OD of the samples to the standard curve. The protocols for IL-1 $\beta$ and NF-B assays were similar to those used for IL-6..

\section{Assessment of oxidative stress markers and TGF- $\beta$ in kidney tissue and urine}

TGF- $\beta$ levels in the urine and kidney tissue were determined using ELISA kits (cat. no. DB100; R\&D Systems, Inc., Minneapolis, $\mathrm{MN}$ ), according to the manufacturer's protocol. All measurements were performed in duplicate. Urine samples were collected in metabolic cages and centrifuged for $1 \mathrm{~min}$ at $10,000 \times \mathrm{g}$ before being assayed or aliquoted and stored at $-20{ }^{\circ} \mathrm{C}$. As part of the assay procedure, $50 \mu \mathrm{l}$ of assay diluent was added to each well. Afterward, $50 \mu \mathrm{l}$ of standard, control, or sample was added to each well and incubated for $2 \mathrm{~h}$ at room temperature. After aspiration and washing, $100 \mu \mathrm{l}$ of a polyclonal antibody specific for TGF- $\beta 1$ conjugated to horseradish peroxidase was added to each well and incubated for $2 \mathrm{~h}$ at room temperature. After aspiration and washing, $100 \mu \mathrm{l}$ of substrate solution was added to each well and incubated for $30 \mathrm{~min}$ at room temperature. Then, $100 \mathrm{~L}$ of a stop solution was added, and color changes were measured at $450 \mathrm{~nm}$ using a BioTek ELISA reader. The protocols for measuring TGF- $\beta 1$ levels in kidney tissues were similar to those used for the urine samples.

The markers for oxidative stress, MDA, SOD, and GPx in kidney tissue homogenates were determined using available kits according to the manufacturer's protocol. The MDA assay kit (cat. no. ZB-MDA-96 A) and SOD assay kit (cat. no. ZB-SOD-96 A) were purchased from ZellBio (Ulm, Germany).

The thiobarbituric acid (TBA) method was used to determine MDA levels. The products of lipid hydroperoxide decomposition can condensate with TBA to form red compounds with an absorption peak at $532 \mathrm{~nm}$. SOD measurements were based on the conversion of superoxide anion into hydrogen peroxide and oxygen. Absorbance at $420 \mathrm{~nm}$ was used to quantify SOD activity.

The GPx assay kit (cat. no. RS 506) was obtained from Randox Laboratories Limited, Crumlin, UK. The oxidation of glutathione by cumene hydroperoxide was catalyzed by GPx. In the presence of NADPH and glutathione reductase, oxidized glutathione was converted into a reduced form with a concomitant conversion of NADPH to NADP+. The decrease in absorption was determined at $340 \mathrm{~nm}$ using a spectrophotometer..

\section{Immunohistochemistry (IHC)}

IL-6, IL-1, P53, and NF-кB expressions were evaluated on paraffin-embedded tissues by a standard immunostaining assay. Briefly, xylene and a graded alcohol series were used for deparaffinization and rehydration, respectively. Then the slides were incubated for $30 \mathrm{~min}$ in a blocking reagent containing $1.5 \%$ hydrogen peroxide in methanol. Antigens were retrieved and placed on slides using a microwave protocol. They were then incubated in serum for $30 \mathrm{~min}$ and immunostained with IL-6 (cat. no. sc-28,343; Santa Cruz Biotechnology, Inc.), IL-1 (cat. no. sc-32,294; Santa Cruz Biotechnology, Inc.), P53 (cat. no. sc-81,168; Santa Cruz Biotechnology, Inc.), and NFкB (cat. no. sc-48,366; Santa Cruz Biotechnology, Inc.) primary antibodies for $1 \mathrm{~h}$ at room temperature.

Afterward, the slides were washed with PBS three times and then incubated with secondary antibodies for $30 \mathrm{~min}$. The sections were stained using 3,30-diaminobenzidine (Dako liquid DAB color solution), and the slides were then counterstained with hematoxylin. An Olympus BX51 microscope (Olympus, Tokyo, Japan) was used to visualize the results. Five different microscopic fields were randomly selected from each slide, 
and positive staining within each slide was measured using Image-Pro Plus 6.1. Finally, quantitative analysis was performed in a blinded manner.

\section{Histopathological studies}

The left kidneys of male rats were harvested and immediately fixed in $10 \%$ buffered formalin phosphate $(\mathrm{pH}$ 7.4) for histological tests. The tissue samples were dehydrated by being passed through graded concentrations of alcohol, cleaned in xylene to remove alcohol, incorporated in paraffin, and allowed to harden. Subsequently, $5-\mu \mathrm{m}$ sections of the paraffin blocks were prepared by microtome and left floating in the water bath. These floating kidney sections were then mounted onto microscopical slides, placed into a drying oven at $60{ }^{\circ} \mathrm{C}$, and stained with Harris' hematoxylin and $1 \%$ eosin. Afterward, histological examinations were carried out using light microscopy. All sections were graded for tubular degeneration, tubular necrosis, and tubular cast formation. The severity of these pathological lesions was assigned as follows: score 0 was considered to be normal; score 1 as mild; score 2 as moderate; score 3 as severe..

\section{Data and statistical analyses}

Prism software was used for the statistical analysis. The results are expressed as means \pm SD. All data sets were first tested for normality using the D'Agostino and Pearson omnibus and Brown-Forsythe tests to ensure the data satisfied the criteria of normal distribution and homogeneity of variances, respectively. A one-way ANOVA was used to analyze all datasets with a normal distribution, and homogeneity was evaluated using Tukey's test for post-hoc comparisons. When unequal SDs were detected, Brown-Forsythe and Welch's ANOVA tests were applied, followed by Dunnett's T3 multiple-comparison test. If the data were not normally distributed and for analysing the results of kidney damage, the Kruskal Wallis test with Dunn's post-hoc test were performed. $\mathrm{P}$ values less than 0.05 were considered statistically significant..

\section{Results}

Effect of different treatments on water intake, urine output, and renal toxicity markers

Water intake and urine output were checked one week after DOX injection (Table 1). Higher water intake $(\mathrm{p}=$ $0.003)$ and lower urine output $(p<0.001)$ were observed in rats in the DOX group than in the control group. Cotherapy with alamandine significantly decreased the effects of DOX. A comparison with the control group revealed that alamandine alone had a diuretic effect.

One week after the last dose of DOX was administered, serum albumin was significantly lower $(p=0.002)$ than in the control group, and urinary albumin was significantly higher $(p<0.001)$. These changes in urinary albumin were significantly $(p<0.001)$ reduced when alamandine was administered $(50 \mathrm{mg} / \mathrm{kg} /$ day) .

DOX administration increased serum BUN $(\mathrm{p}<0.001)$ and serum creatinine $(p<0.001)$ while significantly decreasing urine creatinine $(p=0.033)$. Co-treatment with alamandine significantly reduced serum BUN $(p=0.017)$ and serum creatinine $(p=0.005)$ compared to the DOX treatment. Alamandine co-therapy produced an insignificant increase in urine creatinine $(p=0.405)$.

The results also revealed that creatinine clearance levels were lower in the DOX group than in the control group $(p=0.003)$. Rats co-treated with alamandine exhibited an insignificant increase in creatinine clearance compared to the DOX group ( $p=0.535)$.

Table 1 Effect of treatment with alamandine on water intake, urine output and renal toxicity markers in DOX-treated rats

\begin{tabular}{|c|c|c|c|c|c|}
\hline & Sham & Control & Ala & Dox & Dox + Ala \\
\hline Water Intake (ml/kg/day) & $63.10 \pm 8.68$ & $62.69 \pm 6.90$ & $70.31 \pm 6.68$ & $81.11 \pm 9.84^{* *++}$ & $66.24 \pm 10.21^{\#}$ \\
\hline $\begin{array}{l}\text { Urine output } \\
\text { (ml/kg/day) }\end{array}$ & $27.29 \pm 3.72$ & $27.57 \pm 3.55$ & $34.03 \pm 4.13^{\# \# \# \#^{*}+}$ & $14.39 \pm 4.34^{* * *+t \dagger}$ & $31.20 \pm 4.90^{\# \# \#}$ \\
\hline Serum Creatinine (mg/dl) & $0.71 \pm 0.19$ & $0.65 \pm 0.16$ & $0.67 \pm 0.23$ & $1.61 \pm 0.31^{* * *+t \dagger}$ & $1.06 \pm 0.38^{\# \#}$ \\
\hline $\begin{array}{l}\text { Serum BUN } \\
(\mathrm{mg} / \mathrm{dl})\end{array}$ & $22.81 \pm 4.99$ & $22.98 \pm 5.21$ & $19.22 \pm 6.33$ & $43.45 \pm 13.44^{* * *++t}$ & $28.32 \pm 9.23^{\#}$ \\
\hline Serum Albumin ( $g / d l)$ & $4.06 \pm 0.51$ & $4.32 \pm 0.58$ & $3.96 \pm 0.68$ & $2.34 \pm 0.78^{* *+\dagger}$ & $3.20 \pm 0.81$ \\
\hline $\begin{array}{l}\text { Urine Creatinine } \\
\text { (mg/dl) }\end{array}$ & $76.12 \pm 7.32$ & $76.76 \pm 6.98$ & $81.24 \pm 11.45$ & $58.32 \pm 13.82^{* \dagger}$ & $68.98 \pm 14.34$ \\
\hline $\begin{array}{l}\text { Urine Albumin } \\
\text { (mg/dl) }\end{array}$ & $1.03 \pm 0.21$ & $0.7 \pm 0.19$ & $1.07 \pm 0.24$ & $2.89 \pm 0.36^{* * *+\dagger \dagger}$ & $2.05 \pm 0.39^{\# \# \# * * *+十 \dagger}$ \\
\hline Creatinine Clearance ( $\mathrm{ml} / \mathrm{min})$ & $0.57 \pm 0.14$ & $0.64 \pm 0.17$ & $0.90 \pm 0.53$ & $0.08 \pm 0.03^{* *+}$ & $0.40 \pm 0.18$ \\
\hline
\end{tabular}

Data are expressed as mean \pm SD; $n=7$ for each group

${ }^{+} P<0.05,{ }^{++} P<0.01,{ }^{+++} P<0.001$ compared to the sham;

${ }^{*} P<0.05,{ }^{* *} P<0.01,{ }^{* * *} P<0.001$ compared to the control;

${ }^{\#} P<0.05,{ }^{\# \#} P<0.01,{ }^{\# \#} P<0.001$ compared to the DOX group 
Table 2 Effect of treatment with alamandine on inflammatory cytokines and NF-kB in DOX-treated rats

\begin{tabular}{|c|c|c|c|c|c|}
\hline & Sham & Control & Ala & Dox & Dox + Ala \\
\hline $\begin{array}{l}\text { TGF- } \beta \text { in Urine } \\
\text { (pg/mg creatinine) }\end{array}$ & $16.48 \pm 5.69$ & $17.20 \pm 5.50$ & $23.34 \pm 8.44$ & $79.89 \pm 15.78^{* * *++\dagger}$ & $56.22 \pm 13.33^{* * * \# \#+\dagger+}$ \\
\hline $\begin{array}{l}\text { TGF- } \beta \text { in Kidney } \\
\text { (pg/mg protein) }\end{array}$ & $142.7 \pm 45.99$ & $135.9 \pm 22.56$ & $179.1 \pm 67.20$ & $534.4 \pm 99.90^{* * *++\dagger}$ & $404.4 \pm 77.23^{* * * \# \#+十 \dagger}$ \\
\hline Serum IL-1 $\quad(p g / m l)$ & $363.4 \pm 99.9$ & $350.0 \pm 86.8$ & $342.2 \pm 89.99$ & $552.1 \pm 109.3^{* *+\dagger}$ & $453.9 \pm 105.3$ \\
\hline Serum IL-6 (pg/ml) & $22.32 \pm 5.53$ & $20.10 \pm 5.34$ & $21.23 \pm 6.56$ & $47.46 \pm 10.32^{* * * *+十}$ & $34.98 \pm 9.77^{* * \#+\dagger}$ \\
\hline Serum NF-kB (ng/ml) & $6.5 \pm 2.1$ & $6.3 \pm 1.9$ & $6.4 \pm 1.9$ & $10.2 \pm 2.2^{* \dagger}$ & $7.3 \pm 2.1$ \\
\hline
\end{tabular}

Data are expressed as mean \pm SD; $n=7$ for each group

${ }^{+} P<0.05,{ }^{++} P<0.01,{ }^{+++} P<0.001$ compared to the sham;

${ }^{*} P<0.05,{ }^{* *} P<0.01,{ }^{* * *} P<0.001$ compared to the control;

${ }^{\#} P<0.05,{ }^{\# \#} P<0.01$ compared to the DOX group

\section{Effect of different treatments on inflammatory cytokines and NF-kB}

DOX administration increased the serum levels of IL-1 $\beta$ $(p=0.005)$, IL-6 $(p<0.001)$ and NF-kB $(p=0.011)$. Alamandine co-therapy resulted in lower IL-6 levels when compared to therapy using DOX alone $(p=0.040)$. Insignificant reductions were observed in IL- $1 \beta$ and NF-B levels ( $p=0.439$ and $p=0.091$, respectively).

TGF- $\beta$ levels in the kidney and urine were higher in the DOX group than in the control group $(p<0.001)$. Co-treatment with alamandine decreased these levels $(\mathrm{p}=0.009$ for the kidney and $\mathrm{p}=0.002$ for the urine) (Table 2).

\section{Effects of different treatments on renal oxidative stress markers}

DOX administration markedly increased kidney MDA $(p=0.005)$. The reduction induced by alamandine Cotherapy was not statistically significant $(p=0.999)$. DOX administration caused a significant decrease in kidney SOD $(p=0.006)$ and a significant decrease in renal GPx activity $(p=0.004)$ relative to rats in the control group. Alamandine co-therapy insignificantly diminished these differences $(p=0.554$ for kidney SOD and $p=0.999$ for GPx activity) (Table 3).

\section{Immunohistological study}

All immunohistochemical sections of kidney tissues are presented in Fig. 1. The minimum expressions of IL-6, IL-1, P53, and NF-kb were seen in the control group.
Moreover, the expression levels of P53 were negligible in all groups. Intense staining of IL-6, IL-1, and NF-kb was observed in the DOX group $(\mathrm{p}<0.01)$.

However, the expression of pro-inflammatory cytokines (IL-1, IL-6, and NF-kb) was lower in the alamandine + DOX treatment group than in the DOX group. The results obtained from the alamandine group were similar to those of the control group, except for the finding that IL-1 expression was significantly higher in the alamandine group (Fig. 1).

\section{Histopathological study}

All hematoxylin and eosin (H\&E) stained kidney sections from different experimental groups were evaluated histologically. The kidney sections of the alamandine group did not show any significant histopathological changes However, the histopathological evaluation of the kidneys of DOX-treated animals showed severe tubular degeneration, tubular cast and moderate tubular necrosis. Alamandine co-therapy decreased these pathological changes (Fig. 2).

\section{Discussion}

DOX is used to treat multiple solid tumors but has severe adverse effects on the kidneys $[25,26]$. The mechanisms by which DOX causes glomerular toxicity have are not yet fully understood. However, previous reports have shown that reactive oxygen species and free radicals are the most significant contributors to DOXinduced nephrotoxicity [26-28]. The conversion of DOX

Table 3 Effect of treatment with alamandine renal oxidative stress markers in DOX-treated rats

\begin{tabular}{llcccc}
\hline & Sham & Control & Ala & Dox & Dox + Ala \\
\hline Kidney SOD (U/g tissue) & $71.90 \pm 10.62$ & $74.42 \pm 9.62$ & $69.51 \pm 11.96$ & $48.52 \pm 14.93^{* *+}$ & $59.04 \pm 16.09$ \\
Kidney MDA (nmol/g tissue) & $4.44 \pm 1.53$ & $4.46 \pm 0.94$ & $5.52 \pm 2.61$ & $17.3 \pm 5.31^{* *+\dagger}$ & $14.87 \pm 5.36^{*+}$ \\
$\begin{array}{l}\text { Kidney GPx Activity } \\
\text { (u/mg protein) }\end{array}$ & $11.17 \pm 2.81$ & $11.72 \pm 2.09$ & $10.26 \pm 2.51$ & $4.91 \pm 2.89^{* *+}$ & $6.61 \pm 2.88$ \\
\hline
\end{tabular}

Data are expressed as mean \pm SD; $n=7$ for each group

${ }^{\dagger} P<0.05,{ }^{+\dagger} P<0.01$ compared to the sham;

${ }^{*} P<0.05,{ }^{* *} P<0.01,{ }^{* * *} P<0.001$ compared to the control 


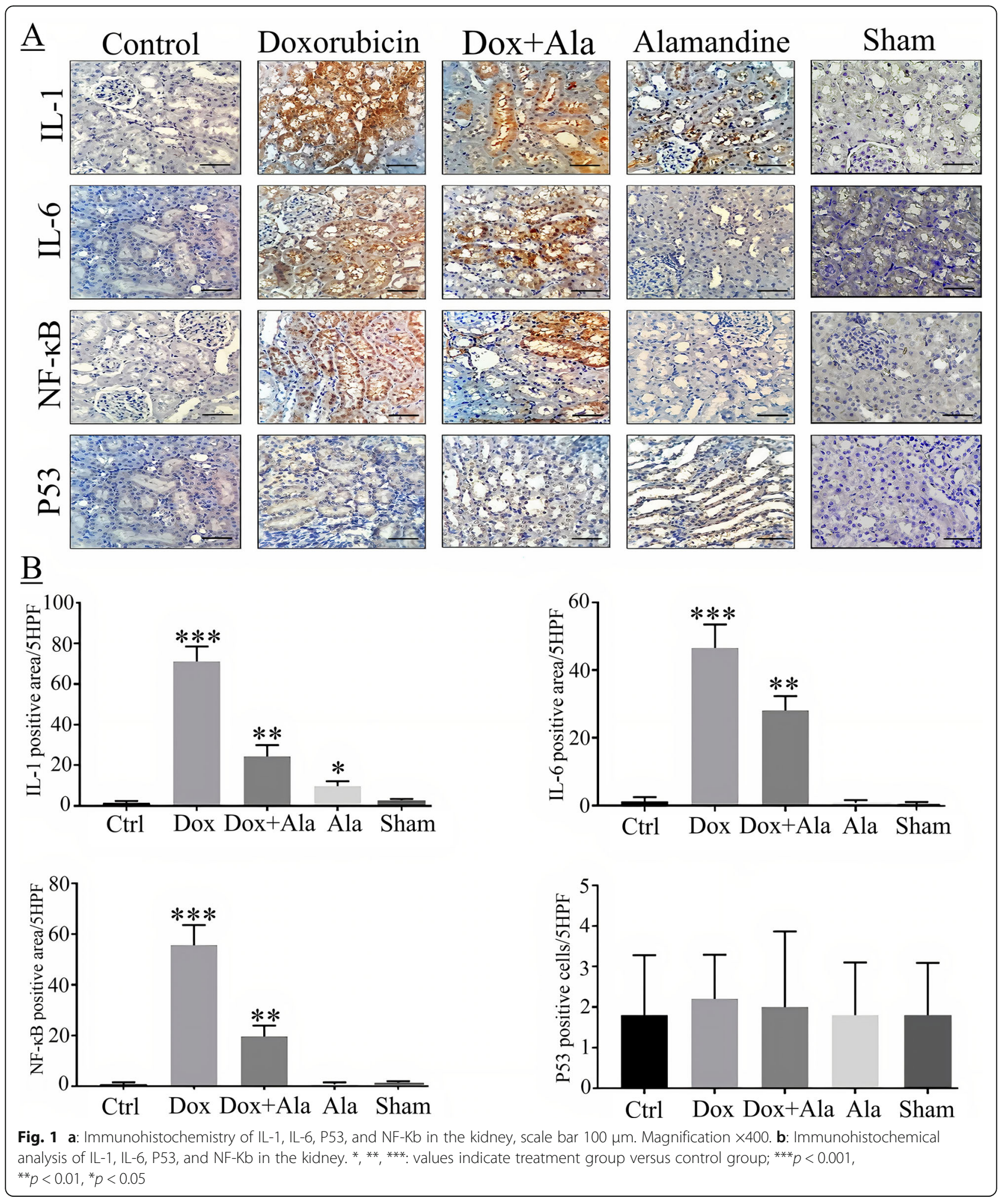

to its semiquinone is thought to play an essential role in its nephrotoxic actions[29]. Semiquinone is unstable under aerobic conditions and, therefore, reacts with molecular oxygen to form superoxide anion radicals[30]. As the number of primary free radicals increases, locally infiltrating neutrophils and active mesenchymal glomerular cells generate more free radicals that damage kidney tissues [31]. DOX exerts direct toxic damage to the 


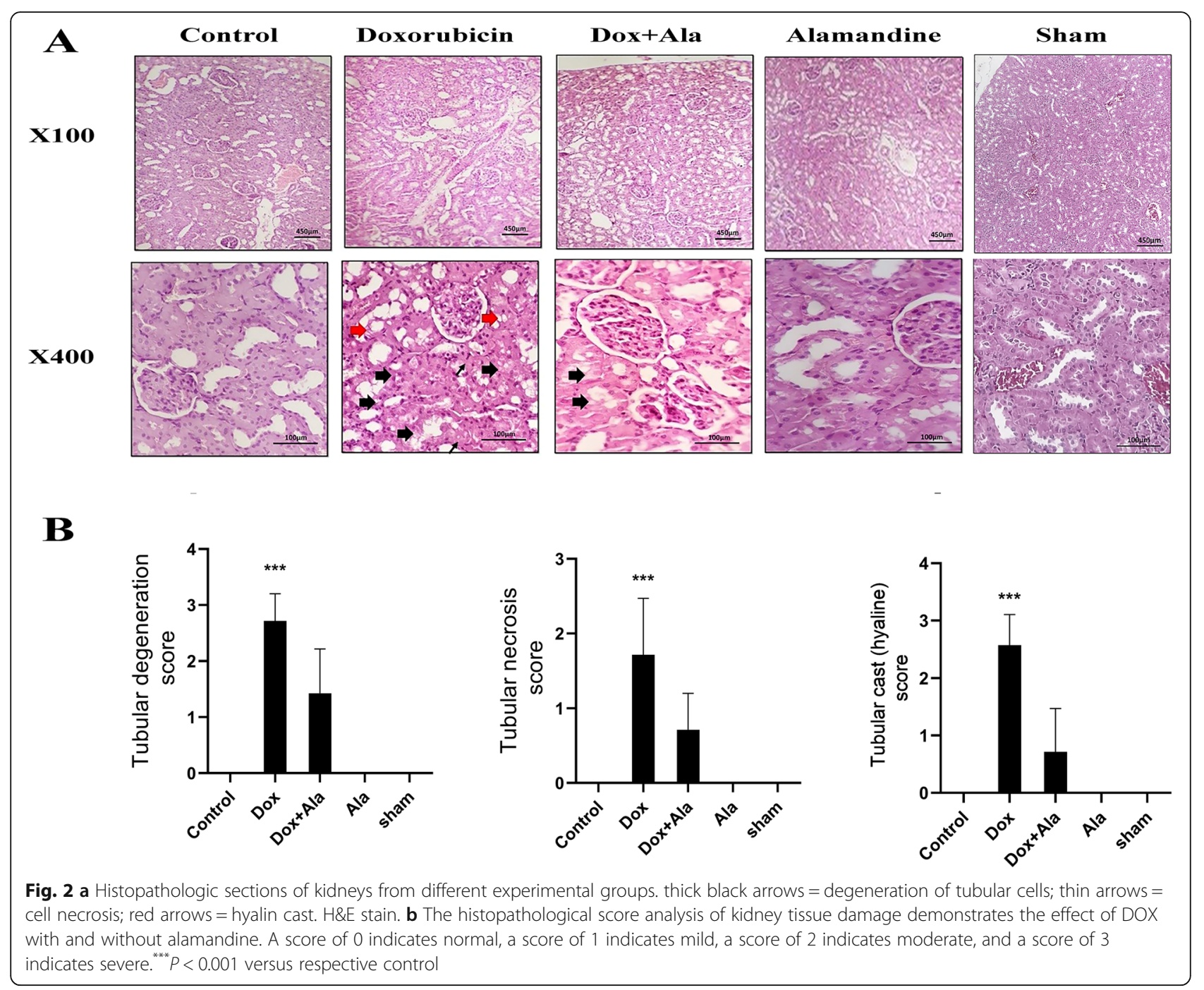

glomerular base membrane, podocytes, and glomerular endothelial cells, inducing tubular interstitial inflammation and fibrosis[32]. As a result, renal function is compromised following DOX administration-in turn, serum urea and creatinine concentrations increase while serum albumin, urea, and creatinine clearance decrease, ultimately leading to extreme proteinuria [33, 34].

In the current research, we used a cumulative dose of DOX injections to induce an experimental nephrotic syndrome model. This model was characterized by albuminuria, hypoalbuminemia, increased serum levels of BUN and creatinine (two significant indicators of renal function), and decreased creatinine clearance) indicator of GFR), all of which are associated with increased oxidative stress and inflammatory factors.

The toxic effects of DOX administration observed in the current study are consistent with the findings of previous studies in which renal function parameters, including serum urea and creatinine, increased [35, 36].
However, administering almandine before, during, and after DOX injections yielded improvements in renal function parameters, including attenuated serum urea, creatinine, albumin, and creatinine clearance. These improvements in DOX-induced renal dysfunction have been confirmed by histological and biochemical findings. For example, DOX has been shown to increase oxidative stress in the kidneys, as indicated by increased lipid peroxidation and changes in antioxidants' status [37].

Oxidative stress is crucial to the development of podocyte damage, glomerular sclerosis, and proteinuria [38]. Our results showed that alamandine co-therapy restored the levels of ROS end products (MDA) and ROSpreventing enzymes (SOD and GPx) in the kidneys compared to the control group. Thus, this treatment could relieve renal oxidative stress in rats with DOX-induced nephrotoxicity. Alamandine's protective effects on albuminuria and glomerular basement membrane damage could be partly explained by its antioxidant property. 

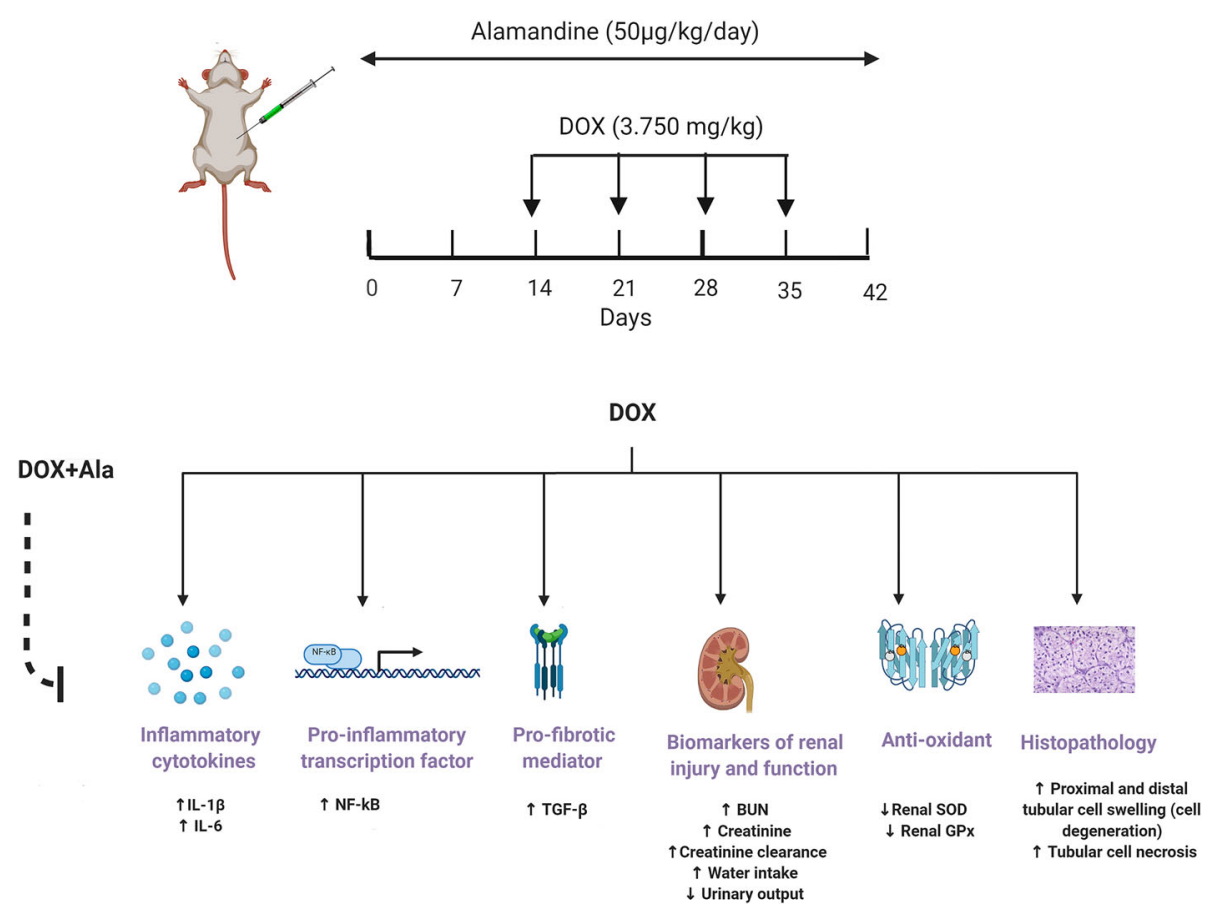

Fig. 3 Effects of alamandine on DOX-induced nephrotoxicity

MDA can indicate lipid peroxidation, and it is used indirectly to assess how much damage has been done to cell membranes [39]. SOD catalyzes the dismutation reaction of superoxide anion to hydrogen peroxide, which is then detoxified into oxygen and water by catalase or glutathione peroxidase. Such proteins prevent damage caused by oxidative stress [40]. The results presented in this research are consistent with those of a previous study indicating that alamandine increased antioxidant protein expression in ventricles exposed to $I / R$ injuries [41].

$\mathrm{NF}-\mathrm{kB}$ is a transcription factor that regulates a wide variety of genes involved in developing renal disease [42, 43]. NF-kB activation plays a pivotal role in the pathogenesis of DOX-induced renal inflammation [44]. NF-kB is responsible for inflammatory reactions via the mediation of TNF- $\alpha$, IL- $1 \beta$, and IL- 6 expressions $[45,46]$. In this study, serum and tissue elevations of TNF- $\alpha$, IL- $1 \beta$, IL-6, and NFKB were observed in DOX-treated rats. Alamandine co-therapy reduced the extent to which $\mathrm{NF \kappa B}$, IL- $1 \beta$, TNF- $\alpha$, and IL- 6 levels were altered by DOX. These results suggest that alamandine has antiinflammatory effects and can reduce the harm done by DOX in harvested tissues.

In this study, alamandine alone increased IL-1. Macrophages have MrgD receptors [47], and it is has been proposed that alamandine binds these receptors, thus increasing IL-1 secretion [47]. However, this increase in IL production requires further investigation.
TGF- $\beta$ was found to be a central mediator of renal fibrosis $[48,49]$. TGF- $\beta_{1}$ can be synthesized by a wide variety of cells, including macrophages, $\mathrm{T}$ and $\mathrm{B}$ fibroblast lymphocytes, and resident renal cells [50]. In the present study, alamandine reduced renal TGF- $\beta_{1}$ levels. TGF- $\beta_{1}$ expression in the kidneys is considered to be the final common pathway leading to structural damage and fibrosis in various glomerular diseases [51, 52]. Urinary TGF$\beta 1$ appears to be a marker of glomerular damage severity. In our study, urinary levels of TGF- $\beta_{1}$ were also increased (and subsequently reduced by alamandine co-therapy).

P53 induction mediates cell apoptosis by activating the caspase-3 protease family and inducing apoptotic cell death [53]. However, in the present study, the expression of P53 IHC did not change in either the DOX or alamandine groups, indicating that DOX side effects are reduced independent of P53. Perhaps other pathways, including ROS, inflammatory cytokines, and $\mathrm{NF}_{\mathrm{K} B} \mathrm{~B}$, work together to damage the kidneys. The elimination of intracellular $\mathrm{H}_{2} \mathrm{O}_{2}$ protects myocytes from DOXinduced apoptosis, probably by inhibiting NF- $\mathrm{kB}$ activation [54]). Thus, alamandine could protect the kidneys by reducing superoxide and blocking the NF-B pathway.

The beneficial effects of alamandine were further illustrated by a histological evaluation using $H \& E$ staining. Histopathologic examinations showed marked pathological lesions. These lesions were characterized by severe proximal and distal tubular cell swelling (cell degeneration), tubular cell necrosis, and a deterioration 
of the architecture of the kidney. Alamandine treatment reduced these pathological lesions.

Surprisingly, the results of this study showed that alamandine alone increased urine volume. Evidence indicates that Ang (1-7) can counterbalance the vascular and tubular action of AngII [55, 56]. Ang (1-7) induces vasodilator, natriuretic, and diuretic effects through the mas receptor [57]. It has been suggested that alamandine, a heptapeptide with an Ang (1-7)-like structure, exhibits actions similar to Ang (1-7) [7, 58-60]. However, alamandine's effects on urine volume require further investigation. Figure 3 is an overview of alamandine's effects on DOX-induced nephrotoxicity.

Both alamandine and Ang-(1-7), owing to their respective receptors Mas and MrgD, are members of the RAS protective arm. This counter-regulatory RAS axis has anti-oxidative, anti-inflammatory, and anti-fibrotic effects. The ACE2/Ang-(1-7)/Mas axis plays a protective role in some renal disease models. A few human studies have revealed the beneficial effect of Ang-(1-7) [61]. However, further studies must be done to elucidate the downstream events triggered by alamandine/MrgD receptors that modulate this protective effect. Understanding the alamandine/MrgD receptor pathway can lead to new pharmacological strategies for repairing the proinflammatory environment that causes various diseases. Current research suggests that drugs that activate this protective arm of the RAS could improve treatments for various disorders, including renal diseases..

Our results suggest that alamandine alleviates DOXinduced nephrotic syndrome in rats, likely owing to its antioxidant and anti-inflammatory properties. These findings indicate that alamandine could be used as a therapeutic agent for DOX-induced nephrotoxicity.

\section{Study limitations}

Our study had several limitations, including the use of pentobarbital at such a high dose $(150 \mathrm{mg} / \mathrm{kg})$ during exsanguination. This dose might have influenced some cytokines and biochemical parameters, such as liver enzymes, urea , and creatinine [62].

Another limitation is that the animals used in this study did not have cancer. Therefore, they did not exhibit all the cellular and molecular changes associated with cancer pathophysiology..

Nevertheless, understanding the mechanisms of DOXinduced nephrotoxicity at an early stage of the disease is important for preventing additional health problems, such as nephrotoxicity, and resulting complications in cancer patients.

\section{Authors' contributions}

A.S.H. and K.J. conceived and planned the experiments. A.S.H., A.CH., and H.A. performed research. A.S.H. and K.J. analyzed data. A.S.H. and K.J. wrote the paper. All authors have read and approved the manuscript.

Funding

There is no funding source.

\section{Availability of data and materials}

The datasets used and/or analyzed during the current study are available from the corresponding author on reasonable request.

\section{Declarations}

Ethics approval

The study was approved by the ethics committee of FUMS (IR.FUMS.REC.1397.014).

Consent for publication

Not applicable.

Competing interests

There is no conflict of interest.

Received: 8 January 2021 Accepted: 23 April 2021

Published online: 29 May 2021

\section{References}

1. Refaie MMM, Amin EF, El-Tahawy NF, Abdelrahman AM. Possible protective effect of diacerein on doxorubicin-induced nephrotoxicity in rats. J Toxicol. 2016;2016:9507563.

2. Lee WW, Harris DC. Adriamycin nephropathy: A model of focal segmental glomerulosclerosis. Nephrology. 2011;16(1):30-8.

3. Rafiee Z, Moaiedi MZ, Gorji AV, Mansouri E. p-Coumaric Acid Mitigates Doxorubicin-Induced Nephrotoxicity Through Suppression of Oxidative Stress, Inflammation and Apoptosis. Arch Med Res. 2020;51(1):32-40.

4. Oktem G, Ayla S, Seckin I, Tanriverdi G, Cengiz M, Eser M, et al. Doxorubicin induced nephrotoxicity: Protective effect of nicotinamide. Int J Cell Biol. 2011;2011:390238.

5. Boute N, Gribouval O, Roselli S, Benessy F, Lee H, Fuchshuber A, et al. NPHS2, encoding the glomerular protein podocin, is mutated in autosomal recessive steroid-resistant nephrotic syndrome. Nat Genet. $2000 ; 24(4): 349-54$.

6. Afsar T, Razak S, Almajwal A, Al-Disi D. Doxorubicin-induced alterations in kidney functioning, oxidative stress, DNA damage, and renal tissue morphology; Improvement by Acacia hydaspica tannin-rich ethyl acetate fraction. Saudi J Biol Sci. 2020;27(9):2251-2260.

7. Lautner RQ, Villela DC, Fraga-Silva RA, Silva N, Verano-Braga T, Costa-Fraga F, et al. Discovery and characterization of alamandine: A novel component of the renin-angiotensin system. Circ Res. 2013;112(8):1104-11.

8. Dong X, Han S kyou, Zylka MJ, Simon MI, Anderson DJ. A diverse family of GPCRs expressed in specific subsets of nociceptive sensory neurons. Cell. 2001;106(5):619-32.

9. Annweiler C, Cao Z, Wu Y, Faucon E, Mouhat S, Kovacic H, et al. Counterregulatory "Renin-Angiotensin" System-based Candidate Drugs to Treat. Infect Disord Drug Targets. 2020;20(4):407-408.

10. Zhu P, Verma A, Prasad T, Li Q. Expression and Function of Mas-Related G Protein-Coupled Receptor D and Its Ligand Alamandine in Retina. Mol Neurobiol. 2020:57(1):513-527.

11. Lu W, Kang J, Hu K, Tang S, Zhou X, Yu S, et al. Angiotensin-(1-7) relieved renal injury induced by chronic intermittent hypoxia in rats by reducing inflammation, oxidative stress and fibrosis. Brazilian J Med Biol Res. 2017; 50(1):e5594.

12. Mori J, Patel VB, Ramprasath T, Alrob OA, Desaulniers J, Scholey JW, et al. Angiotensin 1-7 mediates renoprotection against diabetic nephropathy by reducing oxidative stress, inflammation, and lipotoxicity. Am J Physiol - Ren Physiol. 2014;306(8):F812-21.

13. Rahimi O, Kirby J, Varagic J, Westwood B, Tallant EA, Gallagher PE. Angiotensin-(1-7) reduces doxorubicin-induced cardiac dysfunction in male and female Sprague-Dawley rats through antioxidant mechanisms. Am J Physiol - Hear Circ Physiol. 2020;318(4):H883-H894. 
14. Wang L, Liu C, Chen X, Li P. Alamandine attenuates long-term hypertension-induced cardiac fibrosis independent of blood pressure. $\mathrm{Mo}$ Med Rep. 2019;19(6):4553-4560.

15. Park BM, Phuong HTA, Yu L, Kim SH. Alamandine protects the heart against reperfusion injury via the MrgD receptor. Circ J. 2018;82(10):2584-2593.

16. de Souza-Neto FP, Silva MME, Santuchi MC, de Alcântara-Leonídio TC, Motta-Santos D, Oliveira AC, et al. Alamandine attenuates arterial remodelling induced by transverse aortic constriction in mice. Clin Sci. 2019: 133(5):629-643.

17. Li P, Chen XR, Xu F, Liu C, Li C, Liu H, et al. Alamandine attenuates sepsisassociated cardiac dysfunction via inhibiting MAPKs signaling pathways. Life Sci. 2018;206:106-16.

18. Nematbakhsh M. Renoprotective impact of angiotensin 1-7: Is it certain? Nephropathol. 2019;8(1): e01.

19. Qaradakhi T, Apostolopoulos V, Zulli A. Angiotensin (1-7) and Alamandine: Similarities and differences. Pharmacol Res. 2016;111:820-826.

20. Kilkenny C, Browne WJ, Cuthill IC, Emerson M, Altman DG. Improving bioscience research reporting: The arrive guidelines for reporting animal research. PLoS Biol. 2010;8(6):e1000412.

21. Liu C, Yang C-X, Chen X-R, Liu B-X, Li Y, Wang X-Z, et al. Alamandine attenuates hypertension and cardiac hypertrophy in hypertensive rats. Amino Acids. 2018;50(8):1071-1081.

22. Warpe VS, Mali VR, Arulmozhi S, Bodhankar SL, Mahadik KR. Cardioprotective effect of ellagic acid on doxorubicin induced cardiotoxicity in wistar rats. J Acute Med. 2015:5(1):1-8

23. Iyalomhe GBS, Omogbai EKI, Iyalomhe OOB, Iyalomhe SI. Long-term effects of amlodipine and hydrochlorothiazide combination therapy on creatinine clearance in hypertensive Nigerians. Glob J Pharmacol. 2013;7(1):45-51.

24. Ogundipe DJ, Akomolafe RO, Sanusi AA, Imafidon CE, Olukiran OS, Oladele AA. Effects of two weeks administration of Ocimum gratissimum leaf on feeding pattern and markers of renal function in rats treated with gentamicin. Egypt J Basic Appl Sci. 2016;3(3):219-231.

25. Saad SY, Najjar TA, Al-Rikabi AC. The preventive role of deferoxamine against acute doxorubicin-induced cardiac, renal and hepatic toxicity in rats. Pharmacol Res. 2001;43(3):211-8.

26. Hussain MA, Abogresha NM, AbdelKader G, Hassan R, Abdelaziz EZ, Greish SM. Antioxidant and Anti-Inflammatory Effects of Crocin Ameliorate Doxorubicin-Induced Nephrotoxicity in Rats. Oxid Med Cell Longev. 2021; 2021:8841726

27. Liu LL, Li QX, Xia L, Li J, Shao L. Differential effects of dihydropyridine calcium antagonists on doxorubicin-induced nephrotoxicity in rats. Toxicology. 2007;231(1):81-90.

28. De Beer EL, Bottone AE, Voest EE. Doxorubicin and mechanical performance of cardiac trabeculae after acute and chronic treatment: A review. Eur J Pharmacol. 2001:415(1):1-11.

29. Bachur NR, Gordon SL, Gee M V., Kon H. NADPH cytochrome P-450 reductase activation of quinone anticancer agents to free radicals. Proc Natl Acad Sci U S A. 1979;76(2):954-7.

30. Lee V, Randhawa AK, Singal PK. Adriamycin-induced myocardial dysfunction in vitro is mediated by free radicals. Am J Physiol - Hear Circ Physiol. 1991; 261(4 Pt 2):H989-95.

31. Deman A, Ceyssens B, Pauwels M, Zhang J, Vanden Houte K, Verbeelen D, et al. Altered antioxidant defence in a mouse adriamycin model of glomerulosclerosis. Nephrol Dial Transplant. 2001;16(1):147-50.

32. Jeansson $M$, Björck $K$, Tenstad $O$, Haraldsson B. Adriamycin alters glomerular endothelium to induce proteinuria. J Am Soc Nephrol. 2009;20(1):114-22.

33. Mohebbati R, Khajavi Rad A, Naser Shafei M, Soukhtanloo M, Hosseinian S, Beheshti F, et al. The Effects of Vitamin C on Adriamycin-Induced Hypercholesterolemia in Rat. Curr Nutr Food Sci. 2015;11(4): 309-314.

34. Wu Q, Li W, Zhao J, Sun W, Yang Q, Chen C, et al. Apigenin ameliorates doxorubicin-induced renal injury via inhibition of oxidative stress and inflammation. Biomed Pharmacother. 2021;137:111308.

35. Koul A, Shubrant, Gupta P. Phytomodulatory potential of lycopene from Lycopersicum esculentum against doxorubicin induced nephrotoxicity. Indian J Exp Biol. 2013;51(8):635-45

36. Mancilla TR, Iskra B, Aune GJ. Doxorubicin-induced cardiomyopathy in children. Compr Physiol. $2019 ; 9(3): 905-931$.

37. Al-Harbi MM, Al-Gharably NM, Al-Shabanah OA, Al-Bekairi AM, Osman AMM, Tawfik HN. Prevention of doxorubicin-induced myocardial and haematological toxicities in rats by the iron chelator desferrioxamine. Cancer Chemother Pharmacol. $1992 ; 31(3): 200-4$.
38. Tan RJ, Zhou D, Xiao L, Zhou L, Li Y, Bastacky Sl, et al. Extracellular superoxide dismutase protects against proteinuric kidney disease. J Am Soc Nephrol. 2015;26(10):2447-59.

39. Tacar O, Sriamornsak P. Dass CR. Doxorubicin: An update on anticancer molecular action, toxicity and novel drug delivery systems. Journal of Pharmacy and Pharmacology. J Pharm Pharmacol. 2013;65(2):157-70.

40. Peng CC, Hsieh CL, Wang HE, Chung JY, Chen KC, Peng RY. Ferulic acid is nephrodamaging while gallic acid is renal protective in long term treatment of chronic kidney disease. Clin Nutr. 2012;31(3):405-14.

41. Dobashi K, Ghosh B, Orak JK, Singh I, Singh AK. Kidney ischemia-reperfusion: Modulation of antioxidant defenses. Mol Cell Biochem. 2000;205(1-2):1-11.

42. Sanz AB, Sanchez-Niño MD, Ramos AM, Moreno JA, Santamaria B, RuizOrtega M, et al. NF-KB in renal inflammation. J Am Soc Nephrol. 2010;21(8): 1254-62.

43. Aboyoussef AM, Abdel-Sattar AR, Abdel-Bakky MS, Messiha BAS. Enoxaparin prevents CXCL16/ADAM10-mediated cisplatin renal toxicity: Role of the coagulation system and the transcriptional factor NF-kB. Life Sci. 2021;270: 119120.

44. Sutariya B, Saraf M. a-asarone reduce proteinuria by restoring antioxidant enzymes activities and regulating necrosis factor $\mathrm{KB}$ signaling pathway in doxorubicin-induced nephrotic syndrome. Biomed Pharmacother. 2018:98: 318-324.

45. Vyas D, Laput G, Vyas AK. Chemotherapy-enhanced inflammation may lead to the failure of therapy and metastasis. OncoTargets and Therapy. 2014;7: 1015-23.

46. Somade OT, Ajayi BO, Safiriyu OA, Oyabunmi OS, Akamo AJ. Renal and testicular up-regulation of pro-inflammatory chemokines (RANTES and CCL2) and cytokines (TNF-a, IL-1 $\beta, I L-6)$ following acute edible camphor administration is through activation of NF-kB in rats. Toxicol Reports. 2019;6: 759-767.

47. De Carvalho Santuchi M, Dutra MF, Vago JP, Lima KM, Galvão I, De SouzaNeto FP, et al. Angiotensin-(1-7) and Alamandine Promote Antiinflammatory Response in Macrophages in Vitro and in Vivo. Mediators Inflamm.2019:2019:2401081.

48. Tsakas S, Goumenos DS. Accurate measurement and clinical significance of urinary transforming growth factor-beta. Am J Nephrol.2006;26(2):186-93.

49. Gu YY, Liu XS, Huang XR, Yu XQ, Lan HY. Diverse Role of TGF- $\beta$ in Kidney Disease. Front Cell Dev Biol. 2020:8:123.

50. Kagami S, Border WA, Miller DE, Noble NA. Angiotensin II stimulates extracellular matrix protein synthesis through induction of transforming growth factor- $\beta$ expression in rat glomerular mesangial cells. J Clin Invest. $1994: 93(6): 2431-7$.

51. Border WA, Noble NA. Interactions of transforming growth factor- $\beta$ and angiotensin II in renal fibrosis. In: Hypertension. 1998 ;31(1 Pt 2):181-8.

52. Petreski T, Piko N, Ekart R, Hojs R, Bevc S. Review on Inflammation Markers in Chronic Kidney Disease. Biomedicines. 2021;9(2):182.

53. Jang YM, Kendaiah S, Drew B, Phillips T, Selman C, Julian D, et al. Doxorubicin treatment in vivo activates caspase-12 mediated cardiac apoptosis in both male and female rats. FEBS Lett. 2004 ;577(3):483-90.

54. Wang S, Kotamraju S, Konorev E, Kalivendi S, Joseph J, Kalyanaraman B. Activation of nuclear factor-kB during doxorubicin-induced apoptosis in endothelial cells and myocytes is pro-apoptotic: The role of hydrogen peroxide. Biochem J. 2002:367(Pt 3):729-40.

55. Chappell MC, Diz DI, Yunis C, Ferrario CM. Differential actions of angiotensin-(1-7) in the kidney. Kidney Int Suppl. 1998;68:S3-6.

56. Kuczeriszka M, Kompanowska-Jezierska E, Sadowski J, Prieto MC, Navar LG. Modulating Role of Ang1-7 in Control of Blood Pressure and Renal Function in Angll-infused Hypertensive Rats. Am J Hypertens. 2018 ;31(4):504-511.

57. Sousa-Lopes A, de Freitas RA, Carneiro FS, Nunes KP, Allahdadi KJ, Webb RC, et al. Angiotensin (1-7) inhibits ang II-mediated ERK1/2 Activation by stimulating MKP-1 activation in vascular smooth muscle cells. Int J Mol Cell Med. 2020;9(1):50-61.

58. Soares ER, Barbosa CM, Campagnole-Santos MJ, Santos RAS, Alzamora AC. Hypotensive effect induced by microinjection of Alamandine, a derivative of angiotensin-(1-7), into caudal ventrolateral medulla of 2K1C hypertensive rats. Peptides. 2017:96:67-75.

59. Soltani Hekmat A, Javanmardi K, Kouhpayeh A, Baharamali E, Farjam M. Differences in Cardiovascular Responses to Alamandine in Two-Kidney, One Clip Hypertensive and Normotensive Rats. Circ J. 2017;81(3):405-412.

60. Soltani Hekmat A, Zare N, Moravej A, Meshkibaf MH, Javanmardi K. Effect of prolonged infusion of alamandine on cardiovascular parameters and cardiac 
ACE2 expression in a rat model of renovascular hypertension. Biol Pharm Bull. 2019;42(6):960-967.

61. Santos RAS, Sampaio WO, Alzamora AC, Motta-Santos D, Alenina N, Bader $M$, et al. The ACE2/Angiotensin-(1-7)/Mas axis of the renin-angiotensin system: Focus on Angiotensin-(1-7). Physiol Rev. 2018;98(1):505-553.

62. Mohamed AS, Hosney M, Bassiony H, Hassanein SS, Soliman AM, Fahmy SR, et al. Sodium pentobarbital dosages for exsanguination affect biochemical, molecular and histological measurements in rats. Scientific Reports. 2020 ;10(1):378.

\section{Publisher's Note}

Springer Nature remains neutral with regard to jurisdictional claims in published maps and institutional affiliations.

Ready to submit your research? Choose BMC and benefit from:

- fast, convenient online submission

- thorough peer review by experienced researchers in your field

- rapid publication on acceptance

- support for research data, including large and complex data types

- gold Open Access which fosters wider collaboration and increased citations

- maximum visibility for your research: over $100 \mathrm{M}$ website views per year

At BMC, research is always in progress.

Learn more biomedcentral.com/submissions 